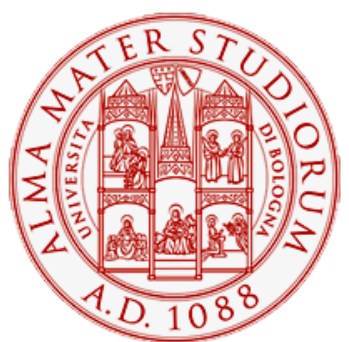

Alma Mater Studiorum - Università di Bologna DEPARTMENT OF ECONOMICS

A Top Dog Tale with Preference Rigidities

Emanuela Randon

Peter Simmons

Quaderni - Working Paper DSE N 839

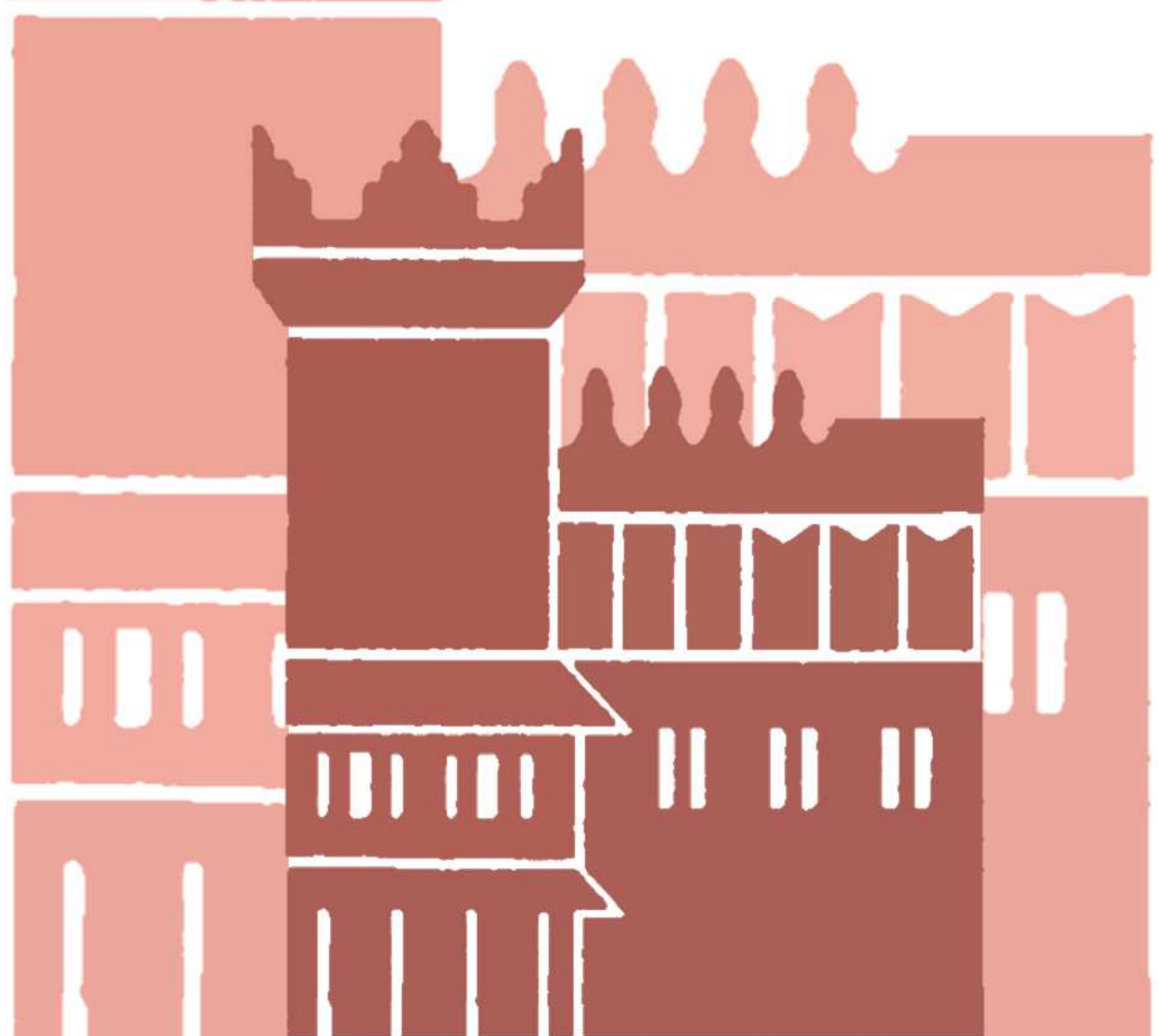




\title{
A Top Dog Tale with Preference Rigidities*
}

\author{
Emanuela Randon ${ }^{\dagger}$ and Peter Simmons ${ }^{\ddagger}$
}

July 1, 2012

\begin{abstract}
With preference rigidities we find Pareto optima of an exchange economy, some of which involve unconsumed endowments. We show that such Pareto Optima can only be attained as market equilibria if there is a top dog in the initial endowment distribution who is richer than the other individuals. The most inegalitarian efficient allocation favouring the top dog is globally stable and is in the core. For endowment distributions with a top dog, the core contains efficient allocations more equal than the market equilibrium. A voting mechanism or government policy can also offset the top dog's power.
\end{abstract}

Keywords: Exchange economy; Complements; Top dog allocation.

JEL classification: D50; D61.

\section{Introduction}

If individuals are price takers, a complete market system can be used to generate efficient outcomes. In an exchange economy, efficiency means both that all goods are consumed and allocational efficiency. Of course, some assumptions are necessary to establish this-typical textbook treatments assume at least local nonsatiation and strict quasiconcavity (Mas-Colell et al (1995)). If preferences exhibit a form of heterogeneous complementarities, then there can be rigidities which prevent the full utilisation of resources. For example, Marie Antoinette perhaps needed wine and cake and had no use for bread, the populace needed bread and wine but without both each was useless. So, one efficient outcome would involve Marie Antoinette consuming all the cake and wine with the bread being thrown away due to the shortage of wine. And the populace starved. Another efficient outcome would involve Marie Antoinette and the populace sharing the available wine and wasting excess amounts of cake and bread. The point is that there are efficient outcomes involving waste, despite some (or all) individuals being nonsatiated in combinations of goods. That is there is "bundled nonsatiation". This surplus of resources is not due to low demand, but occurs because goods

\footnotetext{
${ }^{*}$ We gratefully acknowledge partecipants at ESEM 2010, ASSET 2010, 10th Journées Gerard Varet 2011, II Workshop in General Equilibrium 2011 at University of York and seminar partecipants at University of California San Diego and University of Bologna.

${ }^{\dagger}$ Corresponding author: Department of Economics, University of Bologna. Strada Maggiore 45, 40125 Bologna, Italy. Phone: 0039.051.2092625; Fax: 0039.051.2098040 Email: emanuela.randon@unibo.it

${ }^{\ddagger}$ University of York. Department of Economics. Y010 5DD York. UK. Email: peter.simmons@york.ac.uk
} 
are not available in the right combination to be useful. In such a context, an interesting question is then what prices and what types of initial endowment distributions between individuals will decentralise a particular efficient outcome involving waste as a market equilibrium. Conversely, what is the role of the initial endowment distribution between individuals in reaching different efficient allocations and, under tatonnement, what are the stability properties of these market equilibria? If Marie Antoinette initially owns most of the wine, the market equilibrium will be likely to lead to the efficient outcome in which the populace starves. But if initial ownership of goods is more equal, then the market allocation will lead to more equal consumption of goods useful to the different groups. The endowment distribution plays a crucial role in determining the equity properties of a decentralised Pareto optimum. In some cases markets can leave some individuals with no feasible choices. These individuals are excluded from trading. This can have economic and social cohesion impacts. For example the 2011 unrest in the UK has been ascribed by some to the exclusion of individuals from markets (Bauman, 2011). We start analysing these issues in an exchange economy with cyclical preferences and perfect complements (Scarf, 1960; Hirota, 1981; Anderson et al, 2004). Next generalisations follow. We extend the analysis to environments in which preference rigidities are defined on composite or aggregate goods which need $n$ inputs to be produced and situations in which preferences rigidities are required to reach a subsistence level necessary to activate utility without restrictions on the functional form.

In the benchmark scenario, each good enters the preferences of two individuals and each individual gets utility only from two goods, but no pair of individuals care about exactly the same goods. Each individual wants to consume combinations of goods, "packages" of goods, in which the goods are in fixed proportions. But the set of goods each individual desires overlaps just partially, e.g. any two of the individuals have something in common but not everything. Typical examples can be found in the household environment when individuals are sharing something but not everything. Or in an international trade scenario where countries specialise on a set of goods that only partially overlaps with the set of goods of the other countries. The original motivation of this setting was to highlight the possibility of global instability of general equilibrium (Scarf, 1960) and the theoretical analysis has been confined to that. Here we argue that these environments can be important from a normative and empirical point of view.

As well as a market exchange economy between consumers, there are other scenarios more rooted in public economics in which preference rigidities matter and other ways in which such 
allocations can be realised, e.g. voting or bargaining. A natural application of this setting is in social contracting, when the players are the representative of social groups or social classes and the goods are local public goods or private provided public goods. Since the total amount of resources is fixed, the task is to choose how to share a fixed amount of resources with conflicting interests. For example, the allocation could be of environmental or infrastructure variables such as local public services, carbon permits, vouchers, etc. If each social group has a pool of priorities that only partially overlaps with the pool of the other groups and the amount of resources is limited, then the setting that we are analysing emerges. Each representative will then seek their preferred priorities of infrastructure variables within the allocation mechanism used. We show that the initial endowment distribution and the degree of inequality in the system are decisive for the outcome and for the selection of the set of priorities that will be implemented.

Previous literature has investigated market equilibrium in this exchange economy with preference rigidities with restrictive initial endowment distributions, leading only to the symmetric equilibrium of equal prices and equal consumption of the desired goods. In Scarf's seminal paper, it is assumed that each individual has only the total endowment of one of the goods that he wants to consume. Later research contributions are still characterised by special endowment restrictions. For example, Hirota (1981) analyses the market equilibrium assuming that the sum of the initial endowment across goods is equal for each individual and coincides with the aggregate endowment. Anderson et al (2004) develop an experimental double auction and allow prices to adjust under a nontatonnement rule, based on the same endowment restrictions as those imposed by Hirota. In all these contributions only the symmetric equilibrium emerges.

In this paper, we provide a full analysis of the efficiency, equilibrium and stability properties of these economies with preference rigidities, allowing for more general endowment distributions. Moreover, we generalise the forms of preferences. The principal findings hold even if preference rigidities are defined on composite goods or subsistence levels necessary to activate freely utility.

Firstly, we characterise the full set of efficient allocations. We show that only three classes of Pareto optima arise. There is a single Pareto optimum in which the efficient allocation exhausts the endowment of all the goods. This coincides with the egalitarian solution, in which all individuals enjoy the same level of utility. In all the other efficient allocations, the endowment of one good is totally or partially wasted-we call these corner allocations. These are the "unfair" equilibria, in which one individual (the top dog) gains a much higher level of utility than the other individuals 
(the second class citizen). In the most unfair but efficient equilibrium, only the top dog is the survivor, and the other individuals are left with zero useful consumption.

In our scenario, preferences are not strictly convex and also not strictly monotone in all goods. Therefore, the second fundamental theorem of welfare economics cannot be easily invoked. With perfect complementarity, the set of prices which decentralise a particular efficient allocation is generally not unique. Because of the cyclical nature of preferences, we are able to define the set of prices and initial endowment distributions which will decentralise each type of Pareto optimum as a market equilibrium. Moreover, we identify the effects of the initial endowment distribution on the decentralisation of the different allocations. Pareto efficient allocations which imply the total exhaustion of all goods can be decentralised if and only if the individuals have similar endowment distributions of the goods. Pareto efficient allocations with resource waste arise in the system if and only if there is a top-dog individual who is in an advantaged position relative to the other citizens. The extreme case of efficient allocation in which only the top dog survives and a good is completely wasted is the only one globally stable.

Note that in our context the emergence of a top dog is due to a combination of preference rigidities and initial endowment inequalities. When this combination occurs, markets are extremely weak and they can not neutralise the top-dog power. The market mechanism is not able to overcome the initial endowment inequality. This source of equilibrium inequality is different from that found in some recent literature. Piccione and Rubinstein (2007), Feldman and Serrano (2006), for example, give a rationale for the emergence of a top dog in equilibrium by assuming that there is an exogenous ranking scheme on individuals which defines the distribution of power among them, allowing the powerful individual to seize the endowment of the bottom ranked people. In our context, no exogenous ranking scheme is necessary but rigidities in preferences are the principal cause of these outcome.

For any given initial endowment distribution, we analyse the core and find it is non-empty despite the absence of local nonsatiation. We show that the standard properties of the core hold under weaker conditions than usual. There is a set of allocations that cannot be blocked by coalitions amongst the three consumers. Even with an initial endowment distribution which would generate a top-dog market equilibrium in which the top dog actively trades, the core contains other more equal utility allocations. An egalitarian government may use transfer power or taxation policies to neutralise the top dog. Majority rule also can completely offset the top-dog position. 
Finally we conduct stability analysis of the market solution under tatonnement.

The paper is organised as follows. After the introduction of the base scenario (Scarf's preferences), we find the three classes of Pareto optima of this economy. In Section 4, we analyse the feasible types of market equilibria. We next define the set of prices and initial endowment distributions that can decentralise the different Pareto optima (Section 5 and 6). The next sections look at the structure of the core and the contrast between voting and market outcomes with these preferences. We conduct a stability analysis of the market equilibria (Section 9). A discussion on generalisation strategies will conclude the paper.

\section{Perfect Complements and Cyclical Preferences}

For the sake of simplicity, we consider the original Scarf economy with perfect complements and cyclical preferences of 3 individuals and 3 goods. In the last section, we show how the results are robust in more general settings. Individual preferences are given by

$$
\begin{aligned}
& u_{1}\left(x_{1}, y_{1}, z_{1}\right)=\min \left\{y_{1}, z_{1}\right\} \\
& u_{2}\left(x_{2}, y_{2}, z_{2}\right)=\min \left\{x_{2}, z_{2}\right\}, \\
& u_{3}\left(x_{3}, y_{3}, z_{3}\right)=\min \left\{x_{3}, y_{3}\right\} .
\end{aligned}
$$

There is an interlocking set of perfect complementarities in preferences between the three goods.

For convenience we set the aggregate endowments of the economy at 1 unit of each good. Obviously, changing the scale of the economy does not affect the nature of the results.

\section{Pareto Optima}

The set of feasible allocations is given by

$$
F=\left\{x, y, z \mid \Sigma x_{h} \leq 1, \Sigma y_{h} \leq 1, \Sigma z_{h} \leq 1, x \geq 0, y \geq 0, z \geq 0\right\}
$$

where $x=\left(x_{1}, x_{2}, x_{3}\right)$, etc. The set of efficient allocations are most easily shown in terms of the efficient utility distributions. Define

$$
\begin{aligned}
& P_{1}=\left\{x, y, z \mid(x, y, z) \varepsilon F, u_{1}\left(x_{1}, y_{1}, z_{1}\right)=1-a, u_{2}\left(x_{2}, y_{2}, z_{2}\right)=a, u_{3}\left(x_{3}, y_{3}, z_{3}\right)=a, 0 \leq a \leq 1 / 2\right\} \\
& P_{2}=\left\{x, y, z \mid(x, y, z) \varepsilon F, u_{1}\left(x_{1}, y_{1}, z_{1}\right)=a, u_{2}\left(x_{2}, y_{2}, z_{2}\right)=1-a, u_{3}\left(x_{3}, y_{3}, z_{3}\right)=a, 0 \leq a \leq 1 / 2\right\}, \\
& P_{3}=\left\{x, y, z \mid(x, y, z) \varepsilon F, u_{1}\left(x_{1}, y_{1}, z_{1}\right)=a, u_{2}\left(x_{2}, y_{2}, z_{2}\right)=a, u_{3}\left(x_{3}, y_{3}, z_{3}\right)=1-a, 0 \leq a \leq 1 / 2\right\} .
\end{aligned}
$$


Thus $P_{1}$ is a set of feasible utility distributions which favour individual 1 , in the sense that, as $a$ varies, $u_{1}$ varies in the interval $(1 / 2,1)$, while $u_{2}=u_{3}$ vary in $(0,1 / 2)$. In this situation, we refer to the most favoured individual as the top dog. Similarly in $P_{2}, P_{3}$ a different individual is favoured. The set of efficient utility distributions is given by

$$
P=P_{1} \cup P_{2} \cup P_{3}
$$

The set of efficient allocations is characterised by three types of Pareto optima. Only the first type exhausts the aggregate feasibility constraint. The other cases imply throwing out totally or partially the endowment of one of the goods.

(a) Class I: no waste. There is a Pareto optimum in which the individuals get equal utility $u_{1}=u_{2}=u_{3}=1 / 2$ :

$$
\begin{aligned}
& y_{1}=z_{1}=1 / 2, \\
& x_{2}=z_{2}=1 / 2, \\
& x_{3}=y_{3}=1 / 2,
\end{aligned}
$$

and none of the goods is wasted.

(b) Class II: the aggregate endowment of one good is partially wasted. There is an infinite number of other efficient utility distributions which can be reached without consuming the total endowment of one of the goods. For example set $u_{1}=u_{2}=a, u_{3}=1-a$. This is attained by consumptions

\begin{tabular}{|l|l|l|l|l|}
\hline & $x_{h}$ & $y_{h}$ & $z_{h}$ & $u_{h}$ \\
\hline$h=1$ & 0 & $a$ & $a$ & $a$ \\
\hline$h=2$ & $a$ & 0 & $a$ & $a$ \\
\hline$h=3$ & $1-a$ & $1-a$ & 0 & $1-a$ \\
\hline Total & 1 & 1 & $2 a$ & \\
\hline
\end{tabular}

So long as $0 \leq a \leq 1 / 2$, these allocations are feasible and they cannot be bettered. There is a surplus of good $z$ available, but it cannot usefully be consumed by either individual 3 (he does not want it) nor by individuals 1 and 2 (since there is no matching remaining amount of their complementary good available). For example, if $a=1 / 4$, individual 3 is the top $\operatorname{dog}$ and $50 \%$ of good $z$ is wasted. Similarly, there are two alternative Pareto optima in which only half of one good is not fully consumed but in which there is a different top dog individual

(c) Class III: the aggregate endowment of one good is totally wasted. This class is characterised by three Pareto optima in which one individual gets the total endowment of two of the goods and 
the third good is just completely wasted:

$$
u_{1}=1 \text { with } y_{1}=z_{1}=1 ; u_{2}=u_{3}=0 .
$$

Here 1 uses all of $Y, Z$ which since these are essential goods for 2,3 means that 2,3 are restricted to the utility associated with zero consumption of the goods they care about.

(a)-(c) above define the only types of Pareto optima. In any Pareto optimum, two of the goods must be fully allocated for consumption-at most one good may have no useful consumption purpose. If two of the goods were not fully allocated, we could raise the utility of the person who wants those two goods by giving them the lower amount of whatever is leftover, so that worthwhile consumption increases.

In Figure 1, we represent the full set of Pareto optima. The apex shows the Pareto optimum in which all individuals get equal utility. The upper boundary of the pyramid shows the other two classes in which one good is totally or partially wasted.

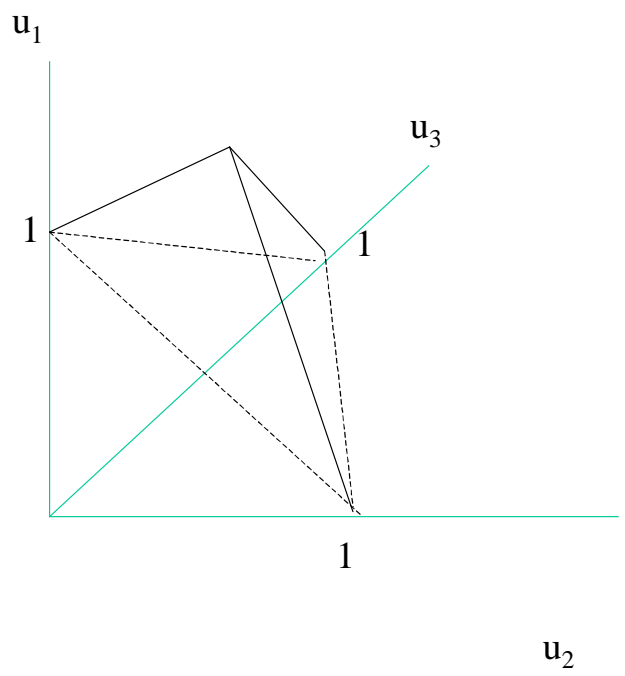

Fig. 1: A graphic representation of the different types of Pareto Optima.

\section{Market Equilibria}

Initial endowments for $h$ are given by $\omega_{h}=\left(X_{h}, Y_{h}, Z_{h}\right)$. Prices are $p=\left(p_{x}, p_{y}, p_{z}\right)$. Note also that homogeneity of degree zero in prices implies that we can impose a price normalisation. The two 
most common are either to set one price equal to unity (but this assumes that any equilibrium will have a positive price in that particular market, i.e. the numeraire good is not in excess supply in equilibrium) or $\Sigma p_{i}=1^{1}$. Here we use the latter normalisation.

All goods are owned by some individual so that, as the aggregate endowment of each good is unity,

$$
\Sigma_{h} X_{h}=\Sigma_{h} Y_{h}=\Sigma_{h} Z_{h}=1
$$

Demands are given by

$$
\begin{aligned}
& f_{x 1}=0, f_{y 1}=f_{z 1}=\frac{p_{x} X_{1}+p_{y} Y_{1}+\left(1-p_{x}-p_{y}\right) Z_{1}}{p_{y}+\left(1-p_{x}-p_{y}\right)} \\
& f_{y 2}=0, f_{x 2}=f_{z 2}=\frac{p_{x} X_{2}+p_{y} Y_{2}+\left(1-p_{x}-p_{y}\right) Z_{2}}{p_{x}+\left(1-p_{x}-p_{y}\right)} \\
& f_{z 3}=0, f_{x 3}=f_{y 3}=\frac{p_{x} X_{3}+p_{y} Y_{3}+\left(1-p_{x}-p_{y}\right) Z_{3}}{p_{x}+p_{y}} .
\end{aligned}
$$

These are continuous in prices for $p_{x}, p_{y}, p_{z}>0$, they satisfy the individual budget constraints with equality and they are homogeneous of degree zero in $p$. Note that they are also continuous at a point at which just one price is zero and the other two prices are positive. However, they are discontinuous at a point at which any two prices are zero.

For a fixed initial endowment distribution between individuals, an equilibrium is a price vector $p$, such that there is no aggregate excess demand $E_{i}$ (with $i=x, y, z$ ), and for any good $i$, if there is excess supply at $p$ of good $i$, then $p_{i}=0$. That is goods which in equilibrium are in excess supply are priced at zero. Formally, for a given initial endowment distribution between individuals, an equilibrium is a set of prices $p_{i}$ and quantities such that

$$
E_{i} \leq 0, p_{i} \geq 0, p_{i} E_{i}=0 \quad i=x, y, z .
$$

Note that an equilibrium of this economy can never have two prices zero. If, for example, $p_{x}=p_{y}=0$, then individual 3 will have an infinite demand for goods $x$ and $y$. Since excess demands are continuous (except where two prices are equal to zero) and they satisfy Walras Law, a competitive equilibrium exists (see, for example, Arrow and Hahn (1971)). In this paper, we analyse whether this equilibrium is unique and stable under tatonnement.

In the next section, we find different combinations of endowments and prices (three different prices, proportional prices, two different prices and all equal prices) which decentralise the equal utility Pareto optimum.

\footnotetext{
${ }^{1}$ By contrast, Scarf used an unusual price normalisation: $\Sigma p_{i}^{2}=1$ which, combined with the non-negativity of prices, means that prices are restricted to the surface of a non-negative quartersphere.
} 


\section{The Decentralisation of Equal Utility Pareto Optimum}

Here we have $u_{h}=1 / 2$ and all goods are consumed. To represent this as a market equilibrium, there must be an initial endowment distribution and prices such that all excess demands are zero (as each good is fully consumed) and prices are all positive.

From Walras law, we can focus on just two excess demands $\left(E_{x}=f_{x 2}+f_{x 3}-1\right.$ and $E_{y}=$ $\left.f_{y 1}-f_{y 3}-1\right)$. In fact to yield the Pareto optimal allocation, we must have $f_{x 2}=f_{y 1}=f_{x 3}=1 / 2$. These equations are not all independent, so we focus on the first two $f_{x 2}=f_{y 1}=1 / 2$. Solving them, we find the price equilibrium levels:

$$
\begin{aligned}
& p_{x}=\frac{\left(Y_{2}-Z_{2}+\frac{1}{4}-Z_{2} Z_{1}-\frac{1}{2} Y_{1}+Y_{1} Z_{2}\right)}{\left(Y_{2} X_{1}-Y_{2} Z_{1}+\frac{1}{2} Y_{2}-Z_{2} X_{1}-\frac{1}{2} Z_{2}+\frac{1}{2} X_{1}-\frac{1}{2} Z_{1}+\frac{1}{4}+Y_{1} Z_{2}-Y_{1} X_{2}+Z_{1} X_{2}\right)} \\
& p_{y}=\frac{\left(\frac{1}{2} X_{2}-Z_{1} X_{2}-\frac{1}{2} X_{1}+Z_{2} X_{1}+\frac{1}{2} Z_{1}-\frac{1}{4}\right)}{\left(Y_{2} X_{1}-Y_{2} Z_{1}+\frac{1}{2} Y_{2}-Z_{2} X_{1}-\frac{1}{2} Z_{2}+\frac{1}{2} X_{1}-\frac{1}{2} Z_{1}+\frac{1}{4}+Y_{1} Z_{2}-Y_{1} X_{2}+Z_{1} X_{2}\right)} .
\end{aligned}
$$

The market equilibrium allocation requires just two equations to be satisfied, whilst there are two normalised prices and six free initial endowment variables that can be selected. So there will be an infinity of ways of decentralising the equal utility efficient allocation.

\subsection{Supporting the equal utility Pareto Optimum with unequal prices}

Here we show that the equal utility Pareto optimum can be decentralised with positive prices iff the endowment of goods for all individuals are collinear, i.e. $\left(X_{h}, Y_{h}, Z_{h}\right)$ lie in a plane. This condition generalises the endowment restrictions used by Hirota and Scarf. Under their restrictions, decentralisation of this Pareto optimum requires equal prices for all goods whereas under our more general restriction this is not necessary. There are infinitely many collinear endowments and relative price vectors which will do the job.

Suppose we take an arbitrary initial endowment distribution $\omega_{1}, \omega_{2}$ with $X_{3}=1-X_{1}-X_{2}, Y_{3}=$ $1-Y_{1}-Y_{2}, Z_{3}=1-Z_{1}-Z_{2}$ :

Proposition 1. The equal utility Pareto optimum is supported by unequal prices iff

$$
\begin{aligned}
\alpha X_{1}+\beta Y_{1}+\gamma Z_{1} & =\kappa, \\
\alpha X_{2}+\beta Y_{2}+\gamma Z_{2} & =\kappa_{1},
\end{aligned}
$$

where $\alpha, \beta$ are arbitrary constants with $0<\alpha \neq \beta \neq \gamma<1$ and $\kappa=(1-\alpha) / 2, \kappa_{1}=$ $(1-\beta) / 2, \gamma=1-\alpha-\beta>0$. 
Proof. See appendix.

This endowment distribution leads to a market equilibrium with prices fixed at the value $p_{x}=\alpha$, $p_{y}=\beta$. Any pair of endowment distributions with the same value of $\alpha$ and $\beta$ will generate the same price equilibrium with the same equal equilibrium utility distribution.

Lemma 1. If (2) holds for some numbers $\alpha, \beta$ then

$$
\alpha X_{3}+\beta Y_{3}+\gamma Z_{3}=(\alpha+\beta) / 2
$$

(2) defines the collinearity restriction between individual endowments which lead to the equal equilibrium utility distribution. We can think of the collinearity restriction as imposing limits on the degree of inequality between the initial endowments of different individuals.

Example 1. The endowment distribution

$$
Z_{1}=0.3 ; Y_{1}=0.7 ; X_{1}=.04 ; Z_{2}=0.35 ; Y_{2}=0.1 ; X_{2}=.59
$$

yields $p_{x}=\alpha=0.28, p_{y}=\beta=0.33$. But the endowment distribution

$$
Z_{1}=0.3 ; Y_{1}=0.4 ; X_{1}=.4 ; Z_{2}=0.35 ; Y_{2}=0.1 ; X_{2}=.59
$$

yields exactly the same equilibrium prices and utility distribution.

We can use (2) to generate special cases of endowment distributions in which the equilibrium prices have special properties. For example, the equal utility distribution can be supported by $p_{y}$ costing twice $p_{x}$ if and only if in $(2) \beta=2 \alpha$. Another case of some interest is that in which in equilibrium goods $x$ and $y$ are equally expensive. Then two individuals trading these goods between themselves would be in a similar position of relative advantage. The equal utility Pareto optimum is supported by $p_{x}=p_{y} \neq p_{z}$ for all goods iff in $(2) \beta=\alpha$.

When $\beta=\alpha$ in (2), the equilibrium prices (1) are

$$
\begin{aligned}
P & =p_{x}=p_{y}=\frac{Z_{3}}{\left(2 Z_{3}+1-X_{3}-Y_{3}\right)}=\frac{Z_{3}}{k+3 Z_{3}}, \\
p_{z} & =1-2 P=\frac{k+Z_{3}}{k+3 Z_{3}},
\end{aligned}
$$

where $k=1-X_{3}-Y_{3}-Z_{3}$. This gives a whole family of values of the initial endowment distributions which generate the market equilibrium giving equal utility for all individuals with $p_{x}=p_{y} \neq p_{z}$. Figure 2 plots these alternative equilibrium prices as a function of $k=1-X_{3}-Y_{3}-Z_{3}$ and $Z_{3}$. 


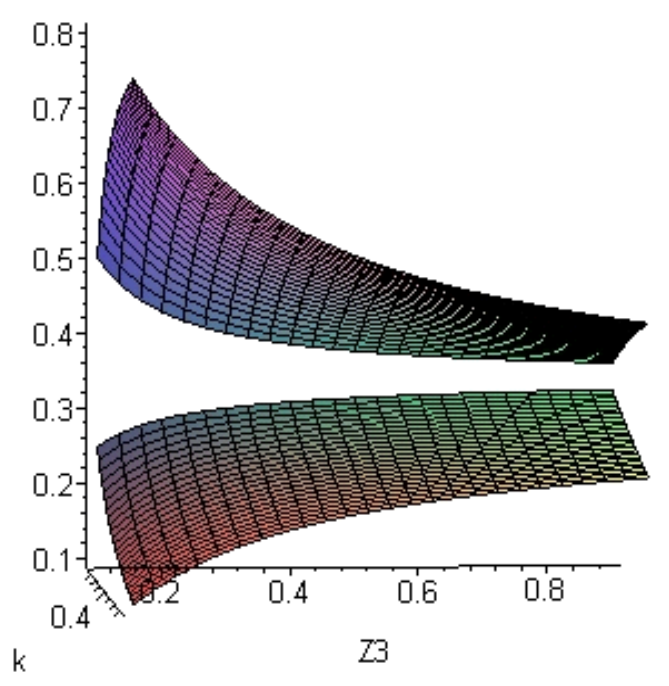

Fig. 2: Equilibrium prices $P, p_{z}$ as a function of $k$ and $Z_{3}$.

For example, if $k=0.2, Z_{3}=0.25$ then $p_{x}=.263, p_{z}=.474$. And so on for other combinations.

\subsection{Supporting the equal utility Pareto Optimum with equal positive prices}

Scarf and Hirota use particular distributions of initial endowments and show that with these, $p_{x}=p_{y}=p_{z}=1 / 3$ gives an equilibrium with equal utilities of $1 / 2$. Hirota's class is defined by

$$
X_{h}+Y_{h}+Z_{h}=1 \quad \text { for all } h
$$

In fact Scarf's endowments, i.e. $Y_{1}=Z_{2}=X_{3}=1$ and all others zero, are a special case of Hirota's class of endowments. Hirota's endowments have the strong interpretation that, when they hold, all individuals have equal wealth if prices are equal for all goods. We can derive this class of endowments from (2) by setting $\alpha=\beta=1 / 3$.

We can then ask what is the full set of initial endowment distributions which make $p_{x}=p_{y}=$ $p_{z}=1 / 3$ a market equilibrium and which leads to the equal utility Pareto optimum.

Proposition 2. The equal utility Pareto optimum is supported by equal positive prices for all goods iff the Hirota conditions hold.

The equilibrium with equal quantity and prices is obtained when the total endowment is equally distributed among individuals. On average, every individual has the same power in contracting since every individual has got a third of the total initial endowment. Setting the prices equal allows one unit of any good to exchange for one unit of any other good. 


\section{Decentralisation of Corner Pareto Optima}

By definition, in a corner Pareto optimum one individual has higher utility than the other two who have equal utility. We refer to the individual who is better off in this Pareto optimum as the top dog. Below we characterise the prices and the exact endowment distribution restriction for each type of corner Pareto optimum. One aspect of the endowment restriction is that the top dog must have a sufficiently large endowment of at least one of the goods which he wishes to consume.

\subsection{Corner Pareto Optima Class II}

Pareto optima Class II have the form $u_{h}=1-a, u_{k}=a=u_{l}$ for $h, k, l=1,2,3$. If we analyse one case say $u_{1}=1-a, u_{2}=a=u_{3}$ the others will follow.

In this case we know that $y_{1}=z_{1}=1-a ; x_{2}=z_{2}=a ; x_{3}=y_{3}=a$ with other consumptions being zero. Generally, we think of 1 as being the favoured individual so that $a<1 / 2$, in which case less than the total endowment of $x$ is consumed at the Pareto optimum. In market terms, prices must be such that $x$ is in excess supply. To decentralise this class of Pareto Optima as a market equilibrium, it must be that $p_{x}=0$. We know that the total endowment of goods $y$ and $z$ is consumed, so in market equilibrium they must exhibit zero excess demand. So we can take $p_{y}, p_{z}>0$ and for example normalise the prices so that $p_{x}+p_{y}+p_{z}=p_{y}+p_{z}=1$.

Proposition 3. Pareto optima with utility distributions $u_{1}=1-a, u_{2}=u_{3}=a$ is supported with prices $p_{x}=0,0<p_{y}=k \neq p_{z}<1$ iff

$$
\begin{aligned}
& k Y_{1}+(1-k) Z_{1}=1-a \\
& k Y_{2}+(1-k) Z_{2}=(1-k) a
\end{aligned}
$$

with $k \neq(1-k)$.

Proof. See appendix.

To support the corner Pareto optima, what matters is the endowment/wealth distribution. In the case above, individual 1 is like a top dog with most of the endowment. The wealth of individuals 2 and 3 valued at the equilibrium prices is lower than the wealth of individual 1 valued at the equilibrium prices, since $a \leq 1 / 2$ and $0<k<1$. Note that although the bottom dogs 2 and 3 have equal equilibrium utility, in general their wealths valued at equilibrium prices differ. If $k=1 / 2$ they have equal wealth, but if $p_{y}=k<1 / 2$ (and so $p_{z}>1 / 2$ ), individual 3 who wants to consume $x$ and $y$ has lower wealth than individual 2, who wants to consume $x$ and $z$. 


\subsection{Corner Pareto Optima Class III}

In this class, the Pareto optimum displays extreme inequity: $u_{1}=1, u_{2}=u_{3}=0$. This can be supported as a market equilibrium only if individual 1 has got all the endowment of the two goods that he likes, whatever the distribution of the good that he does not want among the other individuals. The net-trade conditions in this case for individual 2 and 3 are respectively $k Y_{2}+(1-k) Z_{2}=0$ and $k Y_{3}+(1-k) Z_{3}=0$, which implies that $Y_{2}=Z_{2}=Y_{3}=0$ (since $0<k<1$ and $\left.Y_{h} \geq 0, Z_{h} \geq 0\right)$ and so from the aggregate endowment availability: $Y_{1}=Z_{1}=1$.

Proposition 4. The Pareto optimum with utility distribution $u_{1}=1, u_{2}=u_{3}=0$ is supported with prices $p_{x}=0$, and $p_{y}>0, p_{z}>0$ iff

$$
Y_{1}=Z_{1}=1, Y_{2}=Z_{2}=Y_{3}=Z_{3}=0
$$

In this case the top dog interpretation is extremely inequitable: individuals 2 and 3 have only the endowment of good $x$, that has no value, while individual 1 has wealth 1 again valued at any prices. Only individual 1 survives in the market, individuals 2 and 3 are rationed.

Of course, an egalitarian government can be interested in offsetting the top dog power. Faced with an endowment distribution leading to a top dog outcome, an egalitarian government may wish to use either direct commodity transfers or, failing that, fiscal policy to move to the equal utility efficient outcome. If the government has the power to redistribute goods, it can also just redistribute directly to the equal utility allocation. This will then result in a no trade market equilibrium. Moreover, the government may not have direct redistribution power but does have commodity taxation power. For example, it can impose ad valorem taxes.

\section{The Core}

In this section we prove that the standard properties of the core hold under weaker than normal conditions.

There are some robust properties of the core:

(i) any allocation in the core is Pareto optimal

(ii) any allocation in the core must give each individual a utility level at least as great as that achieved by consuming their initial endowment (individual rationality)

(iii) so long as preferences are at least locally nonsatiated, any competitive equilibrium is in the core. 
In the Scarf economy, we do not have local nonsatiation of preferences: increases in the availability of just one good to an individual will not necessarily increase their utility. Nevertheless properties (i) and (ii) above obviously hold in the Scarf economy.

More interesting, property (iii) also holds in the Scarf economy. This property essentially holds so long as a weak utility increase for all members of a coalition will increase the aggregate cost of the new consumption allocation with goods valued at equilibrium prices. I.e., if $\left(x^{*}, y^{*}, z^{*}\right)$ is a market equilibrium for the given endowment distribution, supported by prices $p$, then for a sub-allocation $(x, y, z)$ to a coalition $S$, if it is true that $u_{h}\left(x_{h}, y_{h}, z_{h}\right) \geq u_{h}\left(x_{h}^{*}, y_{h}^{*}, z_{h}^{*}\right)$ for all coalition members (with strict inequality for at least one member), it means that

$$
p_{x} \sum_{h \in S} x_{h}+p_{y} \sum_{h \in S} y_{h}+p_{z} \sum_{h \in S} z_{h}>p_{x} \sum_{h \in S} x_{h}^{*}+p_{y} \sum_{h \in S} y_{h}^{*}+p_{z} \sum_{h \in S} z_{h}^{*}
$$

The coalition cannot block $x^{*}, y^{*}, z^{*}$ by $x, y, z$. In the Scarf economy, in equilibrium any good which is in excess supply has a zero price and that good is then of no value to the coalition members. So utility increases for coalition members must involve increased consumption of good(s) with a positive price. Hence, the aggregate cost to the coalition of a utility superior allocation must be greater than the cost of the equilibrium allocation to the coalition.

In addition to (i)-(iii), for a given initial endowment distribution, only the subset of feasible Pareto efficient allocations that is not blocked by any of the possible two individual coalitions is in the core. It is easy to show that the condition for an allocation to be unblocked by two person coalitions is that the sum of the initial individual endowments of the good which is commonly desired by the two consumers should be less than the amount of that good available in the allocation. Individual 1 wants to consume $y$ and $z$ in equal proportions, individual $2 \operatorname{good} x$ and $z$, while individual $3 \operatorname{good} x$ and $y$ in equal proportions. An individually rational Pareto efficient allocation $(x, y, z)$ is in the core and it is unblocked by any two individual coalitions if $Y_{1}+Y_{3}<y_{1}+y_{3}$, $Z_{1}+Z_{2}<z_{1}+z_{2}$ and $X_{2}+X_{3}<x_{2}+x_{3}$.

For example, consider individuals 1 and 2 . If $Z_{12}<a$, then neither the single or two person coalition can block the efficient allocation $u_{1}=u_{2}=a$, since also $Z_{1}, Z_{2} \leq Z_{12}$. But if $X_{12}, Y_{12}, Z_{12}>a$, the two person coalition can block this efficient allocation. Similarly, if $Y_{1}, Z_{1}>a$ or $X_{2}, Z_{2}>a$, a single person can block this efficient allocation.

Suppose that the initial endowment distribution leads to a market equilibrium in which 1 is the top dog, so that $p_{x}=0$ and $u_{1}=1-a, u_{2}=u_{3}=a, a<1 / 2$. Consider the efficient utility 
distribution $u_{1}=1-b, u_{2}=u_{3}=b, a<b<1 / 2$. This is in the core (i) since it is Pareto optimal. (ii) it cannot be blocked by any two person coalition since $Y_{1}+Y_{3} \leq 1=1-b+b$, similarly $Z_{1}+Z_{2} \leq 1$. It remains to show that is individually rational. The endowment distribution satisfies $k Y_{1}+(1-k) Z_{1}=1-a$ and, if the top dog actively trades in the market equilibrium, then $Y_{1} \neq Z_{1}$. Suppose $Y_{1}<Z_{1}$, the most that 1 can achieve from autarky is then $u_{1}=Y_{1}<1-a$. Hence, if we choose $b$ so that $Y_{1}<1-b$, then 1 cannot block the utility distribution $1-b, b, b$. Individuals 2 and 3 also cannot block the $b$ allocation. They cannot individually block the market allocation with utility $a$ but, since $b>a$, a fortiori they cannot block they allocation giving them utility $b$.

\section{Application to social choice}

In a social choice context, the three players are the representatives of social groups. The task is to choose how to share a given amount of resources when there are conflicting interests. Indeed, each representative has interests to promote a particular "package" of political interventions that only partially overlaps with the "package" desired by the other candidates. Thus the individual bargaining power is crucial for the selection of the set of social alternatives that will finally emerge. For example, we could think of three regional jurisdictions each of whom has an initial endowment of three different local public goods, e.g. nurses in health delivery, teachers in education or soldiers in defense. Summing the endowments over the jurisdictions gives national endowments for health, education and defence. With a market system, the equilibrium wage rates for different public sector workers and the mix of service provision in the different jurisdictions will emerge. If the resource endowments are such that there is a top dog Walrasian equilibrium, then this jurisdiction will have plentiful provision at the expense of the other two. Generally, this type of equilibrium will involve public sector unemployment of one category of worker and displays inequality. If the allocation is by simple majority voting, the relatively worse off jurisdictions can enforce the equal provision outcome for those services that they value in each jurisdiction. For example, consider the allocation process in which starting from the initial endowment distribution, individuals take turns to propose a new feasible allocation as an alternative to the status quo. If two individuals at least vote in favour, the proposal becomes the new status quo. Then the next individual can propose a new allocation which is voted on against the current status quo. The final allocation if it exists is one which cannot be defeated in majority vote against any new proposal by any individual (Borck, 2007). In this context, this coincides with the equal utility distribution. Suppose that the initial 
endowment distribution is such that the unique market equilibrium utilities generate, say, 3 as a top dog. Then either 1 or 2 can propose the equal utility allocation which will not be defeated in majority vote. And there is no alternative feasible allocation which 3 can propose which will overturn the equal utility outcome in majority vote. Any such allocation must give at least two individuals $u_{h}>1 / 2$, which would require more than one unit of the good which they commonly value. And so cannot be feasible. One region, one vote neutralises the economic power of the resource rich region.

\section{Stability Of Market Equilibria Under Tatonnement}

The original interest in the economy put forward by Scarf was in the stability properties of the equal price equilibrium under a tatonnement rule for price adjustment. Scarf showed that with his particular initial endowment distribution the unique market equilibrium $p_{x}=p_{y}=p_{z}=1 / 3$ corresponding to the Pareto optimum with equal utilities was globally unstable under the price normalisation that he used. Hirota showed that other initial endowment distributions also lead to the equal price equilibrium and that for these other distributions (within the Hirota class but excluding the Scarf case) there was a tendency to local and global stability.

In general, for local stability, the excess demand functions must be downward sloping in their own price and the feedback cross effects between markets should be "small" in comparison with the own price effects. Generally, we can write the Jacobian of the excess demand functions for $x$ and $y$ as

$$
J=\left[\begin{array}{ll}
\partial E_{x} / \partial p_{x} & \partial E_{x} / \partial p_{y} \\
\partial E_{y} / \partial p_{x} & \partial E_{y} / \partial p_{y}
\end{array}\right]
$$

so that

$$
\operatorname{det}(J)=\partial E_{x} / \partial p_{x} \partial E_{y} / \partial p_{y}-\partial E_{x} / \partial p_{y} \partial E_{y} / \partial p_{x}
$$

and $\operatorname{trace}(J)=\partial E_{x} / \partial p_{x}+\partial E_{y} / \partial p_{y}$. If the excess demand functions are downward sloping in their own price then the trace is always negative. The condition for the determinant to be positive (and hence for two eigenvalues whose real parts are negative and local stability) is that

$$
\partial E_{x} / \partial p_{x} \partial E_{y} / \partial p_{y}>\partial E_{x} / \partial p_{y} \partial E_{y} / \partial p_{x}
$$

We can think of this as saying that the aggregate of cross market effects (the LHS) should be small in absolute value relative to the own price effects. 
To explore local stability with an arbitrary initial endowment distribution satisfying (2), we can linearise the excess demand functions around the equilibrium prices $p_{x}=\alpha, p_{y}=\beta$ and compute the trace and the determinant (see Appendix B). In the case equal utilities, the results is ambiguous. Some of these endowment distributions which yield the equal utility equilibrium outcome are locally stable, others are locally unstable even though the equilibrium prices are the same. Instead, in the case of unequal utilities, the Pareto optima class III are globally stable. If one individual is in such a favoured position that he has the total endowment of the two goods he wishes to consume and notrade occurs in the market, then the equilibrium displays strong stability properties. In the other corner Pareto optimum class, still there is a top dog citizen but the difference in terms of wealth with regard to the other citizens is not so remarkable as in the no trade case. The equilibrium that emerges in this case is stable for any initial conditions starting with a zero price for the good which is in excess supply (see Appendix C.1). However starting with arbitrary initial conditions, we show that for equilibria with some trade which have $p_{x}=0$ and individual 1 as the top dog, the sign of the determinant and the trace are ambiguous (see Appendix C.2).

\section{Generalisations and Related issues}

For the sake of simplicity, we have presented the impact of preference rigidities on markets with only three individuals and three goods. In this section we show that such results can be applied to more general settings.

First we can increase the number of individuals and goods pari passu maintaining the cyclic preference complementarities. All the previous results hold in an economy with $n$ individuals and $n$ goods in which each individual wants to consume $n-1$ goods in fixed proportions and the non desired good differs among individuals.

Second similar issues arise if individuals derive utility from the same complementary goods. With just 2 goods and 2 individuals with identical Leontief preferences of the form $u_{h}=\min \left[x_{h}, y_{h}\right]$ if the aggregate endowment of the two goods is unequal then all Pareto optima will involve waste of one of the goods and the only way of decentralising any Pareto optimum will involve the price of the good which is relatively abundant (given consumer preferences) having a zero price. Gale (1960) instead considered a $2 x 2$ economy with identical aggregate endowments of the two goods but with heterogeneous preferences with complementarity. $\left(u_{1}=\min \left[x_{1}, 1 / 2 y_{1}\right], u_{2}=\min \left[x_{2}, 2 y_{2}\right]\right.$. He showed that if there is 1 unit of each good in aggregate then if individuals own only 1 unit of the good 
which is more important to them ( $x$ for 1 and $y$ for 2 ) the excess demand function has a "normal" negative slope whereas if instead 1 owns all $y$ and 2 owns all $x$ the excess demand function has a positive slope. Then under tatonnement the unique interior market equilibrium $\left(x_{1}=1 / 3, y_{1}=\right.$ $2 / 3)$ and $\left(x_{2}=2 / 3, y_{2}=1 / 3\right)$ is unstable. Crocket et al. (2010) run an experiment on the Gale example which gives empirical support to the instability of this unique market equilibrium.

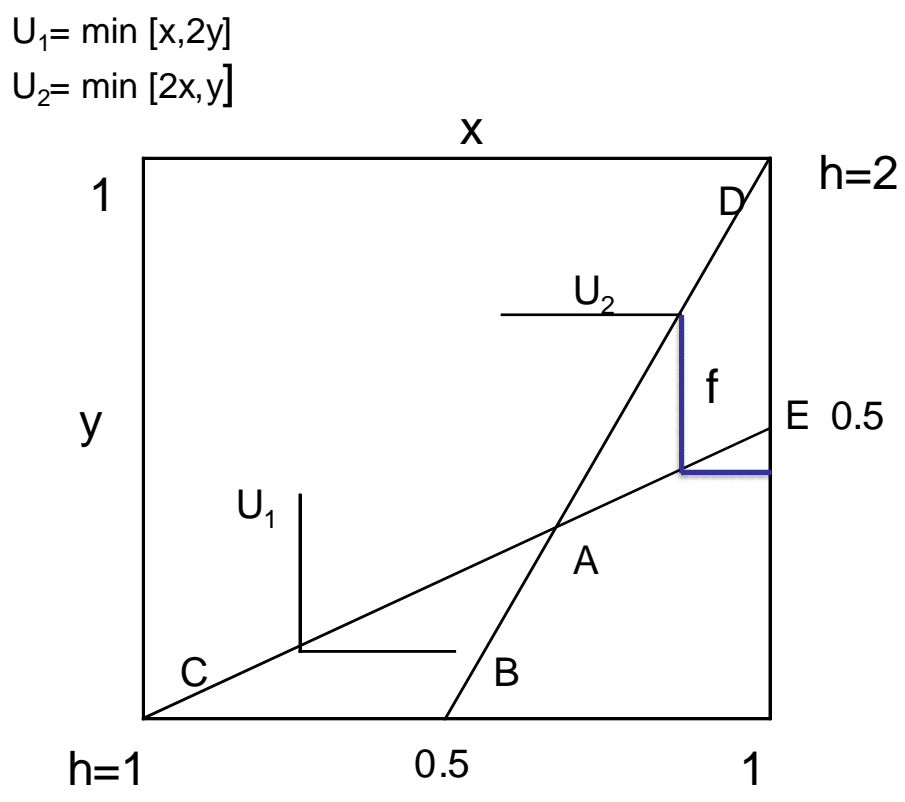

Fig. 3: The area $\mathrm{ABC}$ and $\mathrm{ADE}$ identify the $\mathrm{PO}$ with perfect complements which involve waste of resources.

In fact the set of Pareto optima in the Gale economy includes allocations $x_{h}, y_{h}, h=1,2$ in which each individual could throw away some of their allocation of say $y$ without losing utility. In Figure 3 the set of Pareto optima are defined by the triangles ABC and ADE. Take a Pareto optimum like $f$ at which each individual could throw away part of their allocation of good $y$. An extreme Pareto optimum is at $x_{1}, y_{1}=1, .5$ with $x_{2}, y_{2}=0, .5$. Then individual 2 could throw away his entire endowment of $y$ and not lose utility. Any of these Pareto optima in ADE can be decentralised with a relative price of zero for good $y$. But there will then be no trade in equilibrium, neither individual is prepared to give up any of the good they value. Under tatonnement, points in ADE are all locally stable. Any such Pareto optimum in ADE can be decentralised with $p_{y}=0$ and arbitrary endowments of $y$ but endowments of $x$ at their Pareto optimal level. A local price shock can only make $p_{y}>0$ which will then lead to an excess demand for $x$ and a return to equilibrium so they too are locally stable.

More general results can be obtained in two scenarios. Firstly we can insert a "production" 
setting and we work with composite goods each of which is a function of purchased micro goods. Secondly, it is possible to insert this economy in a more extensive embedding in which individuals have rigidities on a set of goods which constitute a base consumption level (see Randon and Simmons (2010)) necessary to activate utility. Once this subsistence level is reached the utility function can be activated without restrictions on the functional form. A detail analysis of these two cases follows.

\subsection{Rigidities in subsistence consumption levels}

In several situations, individuals need to reach a specific subsistence level before generating any utility. This can happen in situations in which individuals face fixed costs to access markets, or to acquire information. There may also be costs required to achieve a particular life-style. Generally, the set of goods needed to reach these subsistence levels differs from the set of goods that generate utility. The base consumption levels may be characterised by different sets of complementary goods, according to individual needs. For example, living in the country side generally involves a set of complementary goods to reach the work place (i.e. a car, petrol) that differs from individuals living in the city (metro-tickets, newspaper, I-pad).

In such cases, utility $u\left(x_{h v}\right)$ is defined over consumption sets which are given by

$$
\begin{aligned}
& \left\{x_{1 v} \mid \min \left(y_{1}, z_{1}\right) \geq K, x_{1 v} \geq 0\right\} \\
& \left\{x_{2 v} \mid \min \left(x_{2}, z_{2}\right) \geq K, x_{2 v} \geq 0\right\} \\
& \left\{x_{3 v} \mid \min \left(x_{3}, y_{3}\right) \geq K, x_{3 v} \geq 0\right\}
\end{aligned}
$$

The aggregate initial endowments of the goods that form the base consumption levels are $\bar{X}=\bar{Y}=$ $\bar{Z}=1$, while $\overline{X_{v}}$ indicates the vector of initial endowment of the goods that activate utility.

The set of Pareto Optimal allocations for individual 1 is

$$
\begin{aligned}
& \max _{x_{1 v}} u_{1}\left(x_{1 v}\right) \\
\text { st } \min \left(y_{1}, z_{1}\right) \geq & K, x_{1 v} \geq 0 \\
u_{2}\left(x_{2 v}\right) \geq & \bar{u}_{2} \& \min \left(x_{2}, z_{2}\right) \geq K, x_{2 v} \geq 0 \\
u_{3}\left(x_{3 v}\right) \geq & \bar{u}_{3} \& \min \left(x_{3}, y_{3}\right) \geq K, x_{3 v} \geq 0 \\
\Sigma x_{i v} & =\bar{X}_{i v}, i=1 . . n \\
\Sigma x_{i} & =\Sigma y_{i}=\Sigma z_{i}=1
\end{aligned}
$$


The set of Pareto Optimal allocations is identified according to the value of the subsistence level $(K)$. Three different cases can be distinguished:

(1) $K>1 / 2$

Only one individual can meet his subsistence requirements. If individual 1 is the only survivor, i.e. $\min \left(x_{1}, y_{1}\right) \geq K>1 / 2$ then individuals 2,3 have empty consumption sets. At least all of good $z$ is totally wasted, also some of $x, y$, if $1 / 2<K \leq 1$. In that case, only individual 1 will survive and will consume $x_{1 v}=\overline{X_{v}}$. Individual 2 and 3 cannot survive.

(2) $K=1 / 2$

All the three individuals can attain the level $K$, there is no waste and any PO is $\left(x_{1 v}, x_{2 v}, x_{3 v}\right)$ st $\Sigma x_{h v}=\overline{X_{v}}$ and the marginal rate of substitution of the different $x_{v}^{\prime}$ is equated between individuals.

(3) $K<1 / 2$

Again all three individuals can attain $K$. There is no point in them consuming more of $x, y, z$ than will yield $K$ and so excess amounts of these goods are wasted/thrown away. Any PO is $\left(x_{1 v}, x_{2 v}, x_{3 v}\right)$ st $\Sigma x_{h v}=\bar{X}_{v}$ and the marginal rate of substitution of the different $x_{v}^{\prime}$ is equated between individuals.

Next, we look at market solutions.

Suppose that the prices are $p_{x}, p_{y} p_{z}, p_{v}$ and that the individual endowments are $\bar{X}_{h}, \bar{Y}_{h}, \bar{Z}_{h}, \bar{X}_{h v}$ and that $x_{1 v}=\left(x_{11 v}, \ldots, x_{1 n v}\right)$ is a vector of utility generating consumptions for individual 1 .

The individual problem, say for agent 1 is

$$
\begin{aligned}
& \max _{x_{1 v}} u\left(x_{1 v}\right) \\
& \text { st } \min \left(y_{1}, z_{1}\right) \geq K, x_{1 v} \geq 0 \\
& \Sigma p_{1 v} x_{1 v}+p_{y} y_{1}+p_{z} z_{1} \leq \Sigma p_{v i} \bar{X}_{1 v}+p_{x} \bar{X}_{1}+p_{y} \bar{Y}_{1}+p_{z} \bar{Z}_{1}
\end{aligned}
$$

Similarly for the other two individuals. In the appendix (D.1), we show that it is possible to decentralise the different Pareto optimal allocations for $K \leq 1 / 2$ as market solutions. If the subsistence level is not too high $(K \leq 1 / 2)$, well defined Pareto optima exist which can be reached through markets although in many of these $(K<1 / 2)$ the endowment of subsistence necessary goods is so abundant that they are not fully utilised. The equilibrium price for one or more of the subsistence necessary goods is zero in this case. Indeed, the top dog solution corresponds to the 
situation in which the standard of living is so high relatively to the endowment of resources that only the gifted individual survives. In such a case, it is Pareto efficient to ration poor people from markets and waste resources that are useless for the surviving individual. If instead the subsistence level is relatively low, then there are enough resources to allow everybody the access to markets.

\subsection{Rigidities in composite goods}

In this section we show all the previous results hold in a more general context in which micro goods or inputs are required to generate aggregate goods. We can think of home production or at least of combinations of micro goods to yield a utility useful service. For example books $x_{1}$ and tuition $x_{2}$ are purchased to generate education $X\left(x_{1}, x_{2}\right)$; drugs $y_{1}$ and therapy $y_{2}$ yield health $Y\left(y_{1}, y_{2}\right)$ and security alarms $z_{1}$ and fencing $z_{2}$ yield home security $Z\left(z_{1}, z_{2}\right)$. Three individuals may then have Scarf cyclic preferences in education, health and home security. We assume there are three different aggregates and that the "technology" for producing an aggregate quantity is identical for each of three individuals. These technology functions $X\left(x_{1}^{h}, \ldots, x_{m}^{h}\right), Y\left(y_{1}^{h}, \ldots, y_{r}^{h}\right)$ and $Z\left(z_{1}^{h}, \ldots, z_{s}^{h}\right)$ with $h=1,2,3$ are each homogeneous of degree one, concave and increasing with $X(0)=0, Y(0)=0, Z(0)=0$. The goods $x_{j}^{h}$ are traded in competitive markets at prices $p_{i}$, with initial endowment of the micro goods $\bar{x}_{i}^{h}, \bar{y}_{j}^{h}, \bar{z}_{k}^{h}$ (with $i=1, \ldots, m ; j=1, \ldots, r ; k=1, \ldots, s$ and $h=1,2,3)$. Individual utility depends only on the aggregates and in terms of them individuals have cyclical preferences and complements. For consistency reasons we are assuming the same structure of preferences of section 2: individual 1 wants $Y$ and $Z$, individual $2 X$ and $Z$, individual $3 X$ and $Y$. Thus $u_{1}=\min \left[Y\left(y_{1}^{1}, \ldots, y_{r}^{1}\right), Z\left(z_{1}^{1}, \ldots, z_{s}^{1}\right)\right] ; u_{2}=\min \left[X\left(x_{1}^{2}, \ldots, x_{m}^{2}\right), Z\left(z_{1}^{2}, \ldots, z_{s}^{2}\right)\right] ; u_{3}=$ $\min \left[X\left(x_{1}^{3}, \ldots, x_{m}^{3}\right), Y\left(y_{1}^{3}, \ldots, y_{r}^{3}\right)\right]$.

The structure of Pareto optima for this exchange economy of $m+r+s$ goods can be expressed in terms of the aggregates if each of the aggregating functions has constant returns to scale. Despite this, no particular functional restrictions is required for the composition of the inputs. Thus if the aggregate endowments of the individual goods $\bar{x}_{i}=\Sigma_{h} \bar{x}_{i}^{h}$, etc. lead to a square economy in the sense that $X\left(\bar{x}_{1}, \ldots, \bar{x}_{m}\right)=Y\left(\bar{y}_{1}, \ldots, \bar{y}_{r}\right)=Z\left(\bar{z}_{1}, \ldots, \bar{z}_{s}\right)$ then the Pareto optimal utility distributions have the same form as in Scarf's economy with 3 goods. 
The Pareto optimal set is found by solving

$$
\begin{gathered}
\left.\max \min \left[Y\left(y_{1}^{1}, \ldots, y_{r}^{1}\right)\right), Z\left(z_{1}^{1}, \ldots, z_{s}^{1}\right)\right] \\
\text { st } \min \left[X\left(x_{1}^{2}, \ldots, x_{m}^{2}\right), Z\left(z_{1}^{1}, \ldots, z_{s}^{2}\right)\right] \geq K_{2} \\
\min \left[X\left(x_{1}^{3}, \ldots, x_{m}^{3}\right), Y\left(y_{1}^{3}, \ldots, y_{r}^{3}\right)\right] \geq K_{3} \\
\Sigma_{h=1}^{3} x_{i}^{h}=\bar{x}_{i}, \Sigma_{h=1}^{3} y_{i}^{h}=\bar{y}_{i}, \Sigma_{h=1}^{3} z_{i}^{h}=\bar{z}_{i}
\end{gathered}
$$

If the "technologies" are concave, any feasible allocation must involve $\left.X\left(x_{1}^{1}, \ldots, x_{m}^{1}\right)\right)+X\left(x_{1}^{3}, \ldots, x_{m}^{3}\right) \leq$ $X\left(\bar{x}_{i}\right)$, and similarly for $Y, Z$. So the PO must match exactly the three classes of PO previously analysed in Section 3.

How to decentralise them? Take any one of them. Let the goods be traded at prices $p_{i}^{x}, p_{j}^{y}, p_{k}^{z}>0$ so individual $h$ has wealth $\Sigma p_{i}^{x} \bar{x}_{i h}+\Sigma p_{j}^{y} \bar{y}_{j h}+\Sigma p_{k} \bar{z}_{k h}$. Take individual 1 say who wants to $\max$ $\left.\min \left[X\left(x_{1}^{1}, \ldots, x_{m}^{1}\right)\right), Y\left(y_{1}^{1}, \ldots, y_{r}^{1}\right)\right]$ within his budget constraint. Let $C\left(p^{x}, X\right)$ be the total cost function of producing $X$ so that $C\left(p^{x}, X\right)=C^{x}\left(p^{x}\right) X$, which implies $\Sigma p_{j}^{y} y_{j}^{1}=C^{y}\left(p^{y}\right) Y\left(y_{1}^{1}, \ldots, y_{r}^{1}\right), \Sigma p_{i}^{x} z_{i}^{1}=$ $C^{z}\left(p^{z}\right) Z\left(z_{1}^{1}, \ldots, z_{s}^{1}\right)$. Since the whole economy is transformed into an aggregate setting, with quantities $X, Y, Z$ and prices $C^{x}\left(p^{x}\right), C^{y}\left(p^{y}\right), C^{z}\left(p^{z}\right)$ we can apply the earlier results directly. We define $X\left(x_{1}^{h}, \ldots, x_{m}^{h}\right)=\widetilde{X}_{h}, Y\left(y_{1}^{h}, \ldots, y_{r}^{h}\right)=\widetilde{Y}_{h}, Z\left(z_{1}^{h}, \ldots, z_{s}^{h}\right)=\widetilde{Z}_{h}$, and in terms of initial endowments $X\left(\bar{x}_{1}^{h}, \ldots, \bar{x}_{m}^{h}\right)=\bar{X}_{h}, Y\left(\bar{y}_{1}^{h}, \ldots, \bar{y}_{r}^{h}\right)=\bar{Y}_{h}, Z\left(\bar{z}_{1}^{h}, \ldots, \bar{z}_{r}^{h}\right)=\bar{Z}_{h}$, with $h=1,2,3$. For example, the demands for individual 1 are:

$$
\widetilde{Y}_{1}=\widetilde{Z}_{1}=\frac{\Sigma p_{i}^{x} \bar{x}_{i h}+\Sigma p_{j}^{y} \bar{y}_{j h}+\Sigma p_{k} \bar{z}_{k h}}{\left[C^{y}\left(p^{y}\right)+C^{z}\left(p^{z}\right)\right]}
$$

Proposition 5. The equal utility Pareto optimum is supported by unequal prices iff the initial endowment distribution for individual 1 and 2 satisfies the following restrictions

$$
\begin{aligned}
& A(a) \bar{X}_{1}+B(b) \bar{Y}_{1}+C(c) \bar{Z}_{1}=\kappa, \\
& A(a) \bar{X}_{2}+B(b) \bar{Y}_{2}+C(c) \bar{Z}_{2}=\kappa_{1},
\end{aligned}
$$

where $A(a)=A\left(a_{1}, \ldots, a_{r}\right), B(b)=B\left(b_{1}, \ldots, b_{r}\right)$ are arbitrary constants and $\kappa=(B(b)+C(c)) / 2$, $\kappa_{1}=(A(a)+C(c)) / 2$.

Lemma 2. The equal utility Pareto optimum is supported by equal prices iff in (6) $A(a)=$ $B(b)=C(c)$. 
Proposition 6. Pareto optima with utility distributions $u_{1}=1-a, u_{2}=u_{3}=a$ is supported with prices $C^{x}\left(p^{x}\right)=0$ iff

$$
\begin{aligned}
k \bar{Y}_{1}+(1-k) \bar{Z}_{1} & =1-a, \\
k \bar{Y}_{2}+(1-k) \bar{Z}_{2} & =(1-k) a,
\end{aligned}
$$

with $k \neq(1-k)$.

Proposition 7. The Pareto optimum with utility distribution $u_{1}=1, u_{2}=u_{3}=0$ is supported with prices $C^{x}\left(p^{x}\right)=0$, and $p_{y}>0, p_{z}>0$ iff $\tilde{Y}_{1}=Z\left(\bar{z}_{1}^{1}, \ldots, \bar{z}_{r}^{1}\right)=\bar{Y}, Y\left(\bar{y}_{1}^{2}, \ldots, \bar{y}_{r}^{2}\right)=Z\left(\bar{z}_{1}^{2}, \ldots, \bar{z}_{r}^{2}\right)=$ $Y\left(\bar{y}_{1}^{3}, \ldots, \bar{y}_{r}^{3}\right)=Z\left(\bar{z}_{1}^{3}, \ldots, \bar{z}_{r}^{3}\right)=0$.

The sufficient and necessary conditions of these Propositions and Lemma 2 are obtained using the same procedure of Proposition 1-4 (see Appendix A) and simply imposing that $\alpha=A(a)$, $\beta=B(b), \gamma=C(c)$, the prices are $C^{x}\left(p^{x}\right), C^{y}\left(p^{y}\right), C^{z}\left(p^{z}\right)$ and the individual endowment coincides with the endowment of the aggregate goods, i.e. $X_{h}=\bar{X}_{h}, Y_{h}=\bar{Y}_{h}, Z_{h}=\bar{Z}_{h}$.

In equilibria which give unequal utilities, we have to be careful about when marginal cost is zero. With constant returns to scale the most natural assumption is that $C^{I}\left(p^{I}\right)=0$ only if $p^{I}=0$. That is if any micro input price is positive so is the marginal costs of the corresponding aggregate. This would be violated if eg $X()=\Sigma x_{i}$. If the inputs are substitutes, then it is required that only the price of one good is zero.

\section{Conclusion}

Generally in exchange economies with nonsatiated preferences, Pareto efficiency requires the aggregate endowments of each good to be fully consumed. We analyse the case in which, although there is bundled non satiation, Pareto efficiency occurs without the full utilisation of resources. The set of bundled nonsatiated goods that each individual wishes to consume overlaps just partially.

Due to the symmetry of the economy, the obvious Pareto optimum is equal utility for all individuals. In a market economy, we show that this is the equilibrium if individuals have similar initial equal opportunities. But there are many other efficient allocations in which there is a single top dog (Marie Antoinette) and the other two individuals are second class citizens (the populace). We develop an expositary model with three individuals and three goods. Generalisations are then discussed. Particularly we focus on cases in which these preference rigidities are defined on a 
set of goods necessary to access markets and once the requirements are satisfied, the individuals can activate utility without any restrictions. We also consider cases where complementarities are between composite commodities.

Even if the nonsatiation conditions of the fundamental theorem of welfare economics are not strictly satisfied, we show that all types of efficient allocations can be reached as market equilibria for suitable initial endowment distributions. We find the endowment distributions and market equilibrium prices which will decentralise the different Pareto optimum configurations.

When a top dog allocation arises in the market, Pareto efficiency is reached without full use of all the resources. This occurs if in the system there is an individual who is in a favourable endowment position owning the majority of the aggregate endowment of the goods that she wishes to consume but not exactly in the correct proportion and so she trades. After the market trade, she still maintains her privileged position and any coalition between the second class citizens cannot overcome this outcome. The market allocation with this endowment distribution is in the core. With these rigidities in preferences, prices and market trade cannot overcome the basic inequality in the endowment distribution. We show that, when there is a top dog in this sense, the market equilibrium supporting the unequal Pareto optimum has strong stability properties. However, the source of a top dog is essentially in the initial endowment distribution. In this sense, markets cannot serve to offset initial inequalities. Perhaps one consolation is that any individual who is rationed out of markets by prices at least has some company. In such a case, government policy can correct the initial inequality either through direct resource transfer or through commodity based taxation. Another way of overcoming the differential in economic power with such preference rigidities is to allocate consumption bundles with cooperative mechanisms.

\section{References}

[1] Anderson, C. M., Plott, C. R., Shimomura, K. I. and Granat, S. (2004). 'Global Instability in experimental general equilibrium: the Scarf example', Journal of Economic Theory, 115(2), $209-249$.

[2] Arrow, K. J., and Hahn, F. H. (1971). General Competitive Analysis. San Francisco: HoldenDay, Inc. 
[3] Bauman, Z. (2011). 'The London riots: on consumerism coming home to roost', Social European Journal, http:// www.social-europe.eu/2011/08/the-london-ritos-on consumerismcoming home-to-roost/

[4] Borch, R. (2007). 'Voting, Inequality and Redistribution', Journal of Economic Surveys, 21(1), 90-109.

[5] Crockett, S., Oprea R. and Plott, C. (2010). 'Extreme walrasian Dynamics: the Gale example in the Lab. Social Science, Working Paper, 1329, California Institute of Technology, Pasadena, CA. forthcoming in American Economic Review.

[6] Feldman, A. M., and Serrano, R. (2006). Welfare Economics and Social Choice Theory. New York: Springer.

[7] Gale, D. (1963). 'A note of Global Instability of Competitive Equilibria', Naval Research Logistics Quarterly, 10 (1), 81-87.

[8] Hirota, M. (1981). 'On the stability of competitive equilibrium and the patterns of initial holdings: An example' International Economic Review, 22(2), 461-467.

[9] Mas-Colell, A., Whinston, M. D., and Green, J. R. (1995), Microeconomic Theory. New York: Oxford Economic Press.

[10] Piccione, M., and Rubinstein, A. (2007). 'Equilibrium in the Jungle', Economic Jorunal, $117(522), 883-896$.

[11] Randon, E., and Simmons, P. (2010). 'Base consumption levels', Economics Letters, 108(2), $222-224$.

[12] Scarf, H. 1960. 'Some examples of global instability of the competitive equilibrium', International Economic Review, 1(3), 157-172.

\section{A Appendix}

\section{Proof of Lemma 1}

Suppose that:

$$
\begin{aligned}
\alpha X_{1}+\beta Y_{1}+(1-\alpha-\beta) Z_{1} & =(1-\alpha) / 2 \\
\alpha X_{2}+\beta Y_{2}+(1-\alpha-\beta) Z_{2} & =(1-\beta) / 2 .
\end{aligned}
$$


Summing these we obtain

$$
\alpha\left(X_{1}+X_{2}\right)+\beta\left(Y_{1}+Y_{2}\right)+(1-\alpha-\beta)\left(Z_{1}+Z_{2}\right)=1-\alpha / 2-\beta / 2 .
$$

But there is an aggregate endowment of unity of each good so this implies:

$$
\alpha\left(1-X_{3}\right)+\beta\left(1-Y_{3}\right)+(1-\alpha-\beta)\left(1-Z_{3}\right)=1-\alpha / 2-\beta / 2,
$$

and rearranging this we derive:

$$
\alpha X_{3}+\beta Y_{3}+(1-\alpha-\beta) Z_{3}=\alpha / 2+\beta / 2
$$

\section{Proof of Proposition 2 (Case with different prices)}

a) Suppose that the price are unequal and such that: $p_{x}=\alpha, p_{y}=\beta$ and $(1-\alpha-\beta)=p_{z}$, with $0<1-\alpha-\beta<1$, and $0<\alpha \neq \beta \neq \gamma<1$. The equilibrium conditions become:

$$
\begin{aligned}
& f_{y 1}=\frac{\alpha X_{1}+\beta Y_{1}+(1-\alpha-\beta) Z_{1}}{(1-\alpha)}=1 / 2, \\
& f_{x 2}=\frac{\alpha X_{2}+\beta Y_{2}+(1-\alpha-\beta) Z_{2}}{(1-\beta)}=1 / 2,
\end{aligned}
$$

which imply:

$$
\begin{aligned}
\alpha X_{1}+\beta Y_{1}+\gamma Z_{1} & =\kappa, \\
\alpha X_{2}+\beta Y_{2}+\gamma Z_{2} & =\kappa_{1},
\end{aligned}
$$

where $\kappa=1 / 2(1-\alpha), \kappa_{1}=1 / 2(1-\beta)$ and $\gamma=1-\alpha-\beta>0$.

(b) Conversely suppose the conditions (2) hold. Then we have to show that this implies that $p_{x}=\alpha ; p_{y}=\beta$. Again multiplying through (9), we get the linear system:

$$
\begin{aligned}
& p_{x} X_{1}+p_{y} Y_{1}+\left(1-p_{x}-p_{y}\right) Z_{1}=\left(1-p_{x}\right) / 2, \\
& p_{x} X_{2}+p_{y} Y_{2}+\left(1-p_{x}-p_{y}\right) Z_{2}=\left(1-p_{y}\right) / 2
\end{aligned}
$$

Solving these linear equations we get:

$$
\begin{aligned}
p_{y} & =-\frac{\left(-\frac{1}{2} X_{2}+Z_{1} X_{2}+\frac{1}{2} X_{1}-Z_{2} X_{1}-\frac{1}{2} Z_{1}+\frac{1}{4}\right)}{\left(Y_{2} X_{1}-Y_{2} Z_{1}+\frac{1}{2} Y_{2}-Z_{2} X_{1}-\frac{1}{2} Z_{2}+\frac{1}{2} X_{1}-\frac{1}{2} Z_{1}+\frac{1}{4}+Y_{1} Z_{2}-Y_{1} X_{2}+Z_{1} X_{2}\right)}, \\
p_{x}= & \frac{\left(Y_{2}-Z_{2}+\frac{1}{4}-Z_{2} Z_{1}-\frac{1}{2} Y_{1}+Y_{1} Z_{2}\right)}{\left(Y_{2} X_{1}-Y_{2} Z_{1}+\frac{1}{2} Y_{2}-Z_{2} X_{1}-\frac{1}{2} Z_{2}+\frac{1}{2} X_{1}-\frac{1}{2} Z_{1}+\frac{1}{4}+Y_{1} Z_{2}-Y_{1} X_{2}+Z_{1} X_{2}\right)} .
\end{aligned}
$$


This solution requires that the determinant condition

$$
\left(Y_{2} X_{1}-Y_{2} Z_{1}+\frac{1}{2} Y_{2}-Z_{2} X_{1}-\frac{1}{2} Z_{2}+\frac{1}{2} X_{1}-\frac{1}{2} Z_{1}+\frac{1}{4}+Y_{1} Z_{2}-Y_{1} X_{2}+Z_{1} X_{2}\right) \neq 0
$$

should hold.

Recalling the general Hirota conditions (2):

$$
\begin{gathered}
X_{1}=\left(\frac{-\beta Y_{1}-\gamma Z_{1}+\kappa}{\alpha}\right), \\
X_{2}=\left(\frac{-\beta Y_{2}-\gamma Z_{2}+\kappa_{1}}{\alpha}\right),
\end{gathered}
$$

and substituting them in (10) gives $p_{x}=\alpha ; p_{y}=\beta$.

The sufficient and necessary conditions to decentralise the other special cases of of the equal utility distribution with i) $p_{y}$ costing twice $p_{x}$, or ii) $p_{x}=p_{y}$ can be showed simply assuming in the above proof respectively that i) $p_{x}=\alpha, p_{y}=2 \alpha$ and $\alpha\left(X_{1}+2 Y_{1}\right)+\gamma Z_{1}=\kappa, \alpha\left(X_{2}+2 Y_{2}\right)+\gamma Z_{2}=\kappa_{1}$, ii) $p_{x}=p_{y}=\alpha, \alpha\left(X_{1}+Y_{1}\right)+\gamma Z_{1}=\kappa$ and $\alpha\left(X_{2}+Y_{2}\right)+\gamma Z_{2}=\kappa_{1}$.

\section{Proof of Proposition 3 (Case with equal prices)}

The sufficient and necessary conditions are proved using the same procedure of Proposition 2 and imposing $p_{x}=p_{y}=\alpha=\beta=1 / 3$.

\section{Proof of Proposition 4 (Pareto optima Class II)}

(a) In the equilibrium $u_{1}=1-a, u_{2}=u_{3}=a$, which imply excess supply for good $x$. Thus $p_{x}=0$. Suppose that $p_{y}=k$, the equilibrium condition for individual 1 and 2 are:

$$
\begin{aligned}
k Y_{1}+(1-k) Z_{1} & =1-a, \\
\frac{\left(k Y_{2}+(1-k) Z_{2}\right)}{(1-k)} & =a,
\end{aligned}
$$

which imply:

(b) Suppose that (8) hold. We have to show that $p_{y}=k$. The equilibrium condition for individual 1 is:

$$
p_{y} Y_{1}+\left(1-p_{y}\right) Z_{1}=1-a
$$

and 


$$
p_{y}=\frac{\left(a+Z_{1}-1\right)}{\left(-Z_{1}+Y_{1}\right)} .
$$

Substituting (8) of individual 1,

$$
Y_{1}=\left((1-a)-(1-k) Z_{1}\right) / k,
$$

we get:

$$
p_{y}=k \text {. }
$$

\section{B Stability of Equilibrium with Equal Utilities}

The determinant of (5) when $p_{x}=\alpha$ and $p_{y}=\beta$ is equal to

$$
d=\frac{\left(\left(1-2 Z_{2}\right)\left(1-2 Y_{1}\right)+2 Y_{2}\left(1-2 Z_{1}\right)\right)(1-\alpha-\beta)}{2 \alpha(1-\alpha)(\alpha+\beta)(1-\beta)}
$$

whose sign is given by that of $\left(1-2 Z_{2}\right)\left(1-2 Y_{1}\right)+2 Y_{2}\left(1-2 Z_{1}\right)$.

The trace is equal to

$$
\begin{aligned}
t= & -\frac{\left(2 \beta \alpha^{2}+\beta^{2}-2 \alpha^{2}-\beta-\beta \alpha+\alpha\right)}{a(1-a)(a+\beta)(1-\beta)} Y_{1} \\
& -\frac{\left(\beta \alpha+2 \beta^{2}-\alpha^{2}-2 \beta^{2} \alpha-\beta+\alpha\right)}{a(1-a)(a+\beta)(1-\beta)} Y_{2} \\
& -\frac{\left(2 \beta^{2} \alpha-2 \beta^{2}-1+\alpha+3 \beta-3 \beta \alpha\right)}{a(1-a)(a+\beta)(1-\beta)} Z_{2} \\
& -\frac{\left(-2 \beta \alpha^{2}-\beta^{2}+2 \alpha^{2}-1+2 \beta\right)}{a(1-a)(a+b)(1-b)} Z_{1} \\
& -\frac{\left(1-\alpha+2 \beta \alpha+\beta^{2}-2 \beta-\beta^{2} \alpha\right)}{\alpha(1-\alpha)(\alpha+\beta)(1-\beta)},
\end{aligned}
$$

which we can write as

$$
\begin{aligned}
t= & \frac{Y_{1}\left(\beta-\alpha+2 \alpha^{2}\right)}{(\alpha+\beta)(1-\alpha) \alpha}+\frac{Y_{2}\left(\beta-\alpha-2 \beta^{2}\right)}{(1-\beta)(\alpha+\beta) \alpha} \\
& -\frac{(2 \beta-1) Z_{2}}{(\alpha+\beta) \alpha}+\frac{Z_{1}\left(1-\beta-2 \alpha^{2}\right)}{(\alpha+\beta)(1-\alpha) \alpha}-\frac{(1-\beta)}{(\alpha+\beta) \alpha}
\end{aligned}
$$

This is of ambiguous sign. 


\section{Stability with unequal utilities (Class II)}

\section{C.1 Stability for any initial conditions starting with $p_{x}=0$}

In the more general case with equilibrium prices $p_{y}=k, p_{z}=1-k$, the endowment becomes:

$$
\begin{aligned}
& Y_{2}=((1-k) / k)\left(a-Z_{2}\right) ; Z_{3}=(k /(1-k))\left(a-Y_{3}\right), \\
& Y_{1}=1 / k\left[1-a-(1-k) Z_{1}\right], \\
& Z_{1}=1-Z_{2}-Z_{3}, \\
& E_{y}=\frac{\left.\left(p_{y}(1-k)\left(a-Z_{2}\right)\right) / k+\left(1-p_{y}\right) Z_{2}\right)}{\left(1-p_{y}\right)}+\frac{p_{y} Y_{3}+\frac{\left(1-p_{y}\right)\left(a-Y_{3}\right) k}{1-k}}{p_{y}}-1 .
\end{aligned}
$$

Computing its derivative and evaluating at $p_{y}=k$ we obtain

$$
\frac{\partial E_{y}}{\partial p_{y}}=\frac{-2 a+Y_{3}+Z_{2}}{k}<0 .
$$

The equilibrium is always stable since $\left(Y_{3}-a\right)<0$ and $\left(Z_{2}-a\right)<0$.

As an example we know that a Pareto optimum with unequal utilities can be supported as an equilibrium with two equal prices $p_{y}=p_{z}=1 / 2$ when the endowment distribution is:

$$
Y_{1}=2-2 a-Z 1, Y_{2}=a-Z_{2} ; Y_{3}=a-Z_{3}
$$

With this endowment distribution, the excess demand function for $y$ has the form

$$
E_{y}=p_{y}\left(2-2 a-Z_{1}\right)+\left(1-p_{y}\right) Z 1+\frac{p_{y}\left(a-Z_{3}\right)+\left(1-p_{y}\right) Z_{3}}{p_{y}}-1 .
$$

Then we obtain:

$$
\frac{\partial E_{y}}{\partial p_{y}}=2-2 a-2 Z_{1}-\frac{Z_{3}}{p_{y}^{2}} .
$$

We should evaluate it at the equilibrium: $p_{y}=1 / 2$, obtaining:

$$
\frac{\partial E_{y}}{\partial p_{y}}=2-2 a-2 Z_{1}-4 Z_{3}<0 \text {. }
$$

Note that $2\left(1-Z_{1}-Z_{3}\right)=2 Z_{2}$. Thus (14) becomes: $2\left(-a+Z_{2}-Z_{3}\right)<0$ since $Z_{2}-a<0\left(Y_{2}\right.$ cannot be negative). 


\section{C.2 Stability for arbitrary initial conditions}

The determinant of (5) when $p_{x}=k, p_{y}=1-k$ and $Y_{1}=\left(-(1-k) Z_{1}+(1-a)\right) / k ; Y_{2}=$ $\left(-(1-k) Z_{2}+(1-k) a\right) / k$ is

$d=\frac{\left.\left.a\left[2\left(X_{1}+X_{2}\right)(1-k)+k-2\left(1+Z_{2}\right)\right]+2 a^{2}-2(1-k)\left[\left(Z_{2} X_{1}-Z_{1} X_{2}+X_{2}\right)\right]+\left(1-Z_{1}+Z_{2}\right)\right]\right)}{k^{2}(1-k)}$.

The trace is equal to

$$
t=\frac{\left.k^{2}\left(2 a-1+2 X_{2}-2 Z_{2}+X_{1}\right)+k\left(2(1-a)-Z_{1}-X_{2}-X_{1}\right)\right)-1+Z_{1}+Z_{2}}{k^{2}(1-k)} .
$$

The sign of the trace and of the determinant are ambiguous. 


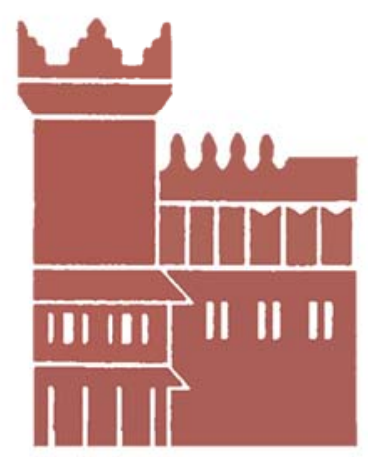

Alma Mater Studiorum - Università di Bologna DEPARTMENT OF ECONOMICS

Strada Maggiore 45

40125 Bologna - Italy

Tel. +39051 2092604

Fax +390512092664

http://www.dse.unibo.it 